\title{
A deeper understanding of VEGFR inhibitors
}

Although vascular endothelial growth factor receptor (VEGFR) tyrosine kinase inhibitors are effective at treating renal cell carcinoma, members of this drug class have different clinical efficacies and sideeffect profiles, indicating that they might not all block VEGFR signalling to an equal extent.

To help understand the clinical differences between VEGFR inhibitors, a team from Pfizer investigated how such inhibitors structurally interact with VEGFR. This was then linked to lipophilicity-based ligand efficiency (LipE; a measure that reflects the potency and lipophilicity of the ligand) and clinical responses.

For their crystallography and in vitro assays, the authors used a VEGFR2 kinase construct that contained the catalytic domain and the juxtamembrane domain (although the latter can contribute to drug potency, many studies have not used this domain). They determined crystal structures of this construct bound to each of five VEGFR inhibitors: axitinib, pazopanib, sorafenib, sunitinib and tivozanib. The results showed that the VEGFR inhibitors bind two distinct juxtamembrane conformations. These were named 'juxtamembrane-in', where the juxtamembrane domain is folded into a portion of the kinase interdomain space called the regulatory domain pocket (RDP), and 'juxtamembraneout' (where the juxtamembrane is not in this space).

Moreover, the binding mode for the VEGFR tyrosine kinase inhibitors identified by these studies is not captured by current classifications. The authors classified these as type IVb inhibitors (pazopanib and sunitinib), which bind the juxtamembrane-in conformation deep into the RDP, and type IVa inhibitors (axitinib), which bind like type IVb inhibitors and have additional hydrogen bond interactions.

Determining the potency for eight VEGFR inhibitors revealed that the order of LipE (highest to lowest) was as follows: axitinib, pazopanib, cediranib, sunitinib, brivanib, tivozanib, linifanib then sorafenib.

Then the authors compared the LipE data to the structural data.

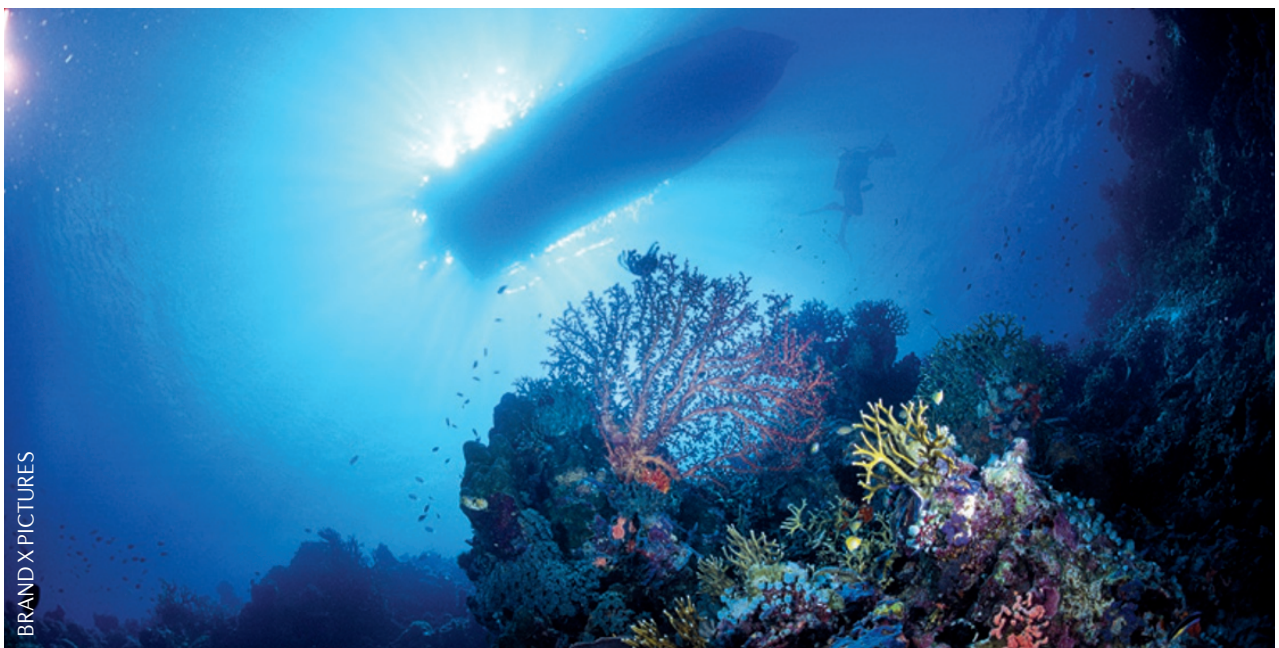

This revealed that for high LipE it was important to have direct hydrogen bond interactions between the inhibitor and the protein that resulted in a fully filled channel that spans from the ATP binding site to the RDP: this type of interaction was complementary to the juxtamembrane-in conformation. Drugs with large substituents that extended into the RDP caused displacement of the juxtamembrane domain (to the juxtamembrane-out conformation) and had lower LipE as a result (linifanib, sorafenib and tivozanib).

The authors then linked LipE to clinical effects by using the clinical end points of progression-free survival and hypertension obtained from clinical trials that used similarly selected patients. Analysis of clinical data against LipE showed positive correlations for cytokine-refractory renal cell carcinomas $(R=0.86)$ and first-line renal cell carcinomas ( $R=0.97$ ), suggesting that the extent of VEGF blockade (determined by LipE) is the factor that determines the different clinical effects of VEGFR inhibitors.

So this study showed that the clinical performance of a VEGFR inhibitor can be linked to its LipE and a defined conformation of VEGFR. The authors highlight that if this technique is to be prospectively applied to aid the design of drugs with optimal efficiency for other target proteins, the regulatory conformations will need to be identified early in the drug discovery process.

Charlotte Harrison

ORIGINAL RESEARCH PAPER McTigue, M. et a Molecular conformations, interactions, and properties associated with drug efficiency and clinical performance among VEGFR TK inhibitors. Proc. Natl Acad. Sci. USA 17 Sep 2012 (doi:10.1073/pnas.1207759109) 